\title{
Understanding stand-to-sit maneuver: Implications for motor system neuroprostheses after paralysis
}

\author{
Sarah R. Chang, BS; ${ }^{1-2^{*}}$ Rudi Kobetic, MS; ${ }^{\mathbf{1}}$ Ronald J. Triolo, $\mathbf{P h D}^{\mathbf{1 , 3}}$ \\ ${ }^{1}$ Louis Stokes Cleveland Department of Veterans Affairs Medical Center, Cleveland, OH; Departments of ${ }^{2}$ Biomedical \\ Engineering and ${ }^{3}$ Orthopaedics, Case Western Reserve University, Cleveland, $\mathrm{OH}$
}

\begin{abstract}
Standing up, standing, and walking functions can be restored to people with spinal cord injury by contracting the paralyzed hip, knee, and ankle muscles with electrical stimulation. Restoring these functions using electrical stimulation requires controlled activation to provide coordinated movements. However, the stand-to-sit (STS) maneuver involves eccentric contractions of the quadriceps to control lowering of the body to the seated position, which is difficult to achieve with stimulation alone and presents unique challenges to lower-limb neuroprostheses. In this study, we examined the biomechanics of the STS maneuver in five nondisabled individuals and five users of an implanted neuroprosthesis. Neuroprosthesis users relied heavily on their upper limbs during STS, with peak supporting forces approximately $25 \%$ body weight, and exhibited an average vertical acceleration at the impact six times higher than that of the nondisabled subjects $(p<0.001)$. Sitting with stimulation resulted in impact forces at initial contact with the seating surface averaging 1.4 times body weight and representing an average of twice the impact forces of the nondisabled subjects $(p<0.001)$. These results indicate a need for additional interventions to better control descent, minimize impact, and gently transition from standing to sitting to achieve a more natural movement and reduce the risk of injury.
\end{abstract}

Key words: biomechanics, functional neuromuscular stimulation, impact force, neuroprosthesis, paralysis, SCI, spinal cord injury, stand-to-sit, upper-limb force, vertical acceleration.

\section{INTRODUCTION}

Neuroprostheses using functional neuromuscular stimulation (FNS) can restore standing and personal mobility for individuals with spinal cord injury (SCI) [12]. Small electrical currents applied to the intact peripheral nerves cause the paralyzed muscles to contract and produce the necessary forces and joint torques to support the body against collapse and generate the movements required for stepping [3-5]. The improved mobility due to stimulation also provides physical and psychological benefits, such as cardiovascular fitness, reduced risk of pressure sores, and an enhanced self-image [6-8]. While the sit-to-stand transition [9], standing and standing balance [10-12], and walking with stimulation [7,13-14] have been studied extensively, the stand-to-sit (STS) maneuver has received relatively little attention. Controlling descent of the body and minimizing impact with the

\footnotetext{
Abbreviations: $\% \mathrm{BW}=$ percent of body weight, AIS $=$ American Spinal Injury Association Impairment Scale, FNS = functional neuromuscular stimulation, OLC $=$ open-loop controller, ONZOFF $=$ ON-ZONE-OFF, SCC $=$ switching curve controller, $\mathrm{SCI}=$ spinal cord injury, STS = stand-to-sit.

*Address all correspondence to Sarah R. Chang, BS; Louis Stokes Cleveland Department of Veterans Affairs Medical Center, APT Center, 10701 East Blvd, 151AW/APT, Cleveland, OH 44106; 216-791-3800, ext 3834; fax: 216-707-6420. Email: sarah.r.chang@case.edu http://dx.doi.org/10.1682/JRRD.2013.12.0264
} 
seating surface are important goals for practical and safe operation of lower-limb neuroprostheses.

One of the main challenges to achieving more natural and safer STS transition is the difficulty in controlling eccentric contractions of the knee extensor muscles with electrical stimulation. The quadriceps muscles need to lengthen smoothly and continuously while being activated with stimulation to control the lowering of the body to the seated position [15-16]. The complex interplay of sensory information related to muscle length, tension, joint position and moment, and acceleration that would normally determine whether contractions of the quadriceps muscles need to be adjusted to control the downward movement of the body during STS are lacking or insufficiently integrated into existing lower-limb neuroprostheses.

A number of controllers have previously been designed to address the need for a controlled STS transition. A low-level, closed-loop switching curve controller (SCC) assisted standing up and sitting down by employing a simple switching curve to modulate stimulation to the quadriceps, depending on whether a prescribed angular velocity at a given knee angle was exceeded during the maneuver [17]. This controller was more effective than an unramped open-loop controller (OLC) that simply turned stimulation off to produce the STS transitions. The peak knee angular velocity for the SCC was 1.7 times greater than that for nondisabled $(170.9 \pm 47.6 \%$ vs $101.7 \pm 29.5 \%$ ). With the OLC, subjects only started to sit down once the stimulation was turned off. As a result, STS was completed without the assistance of FNS and subjects needed to rely solely on upper limbs for support and control of descent. It was also suggested that the OLC could potentially be improved by ramping down the quadriceps stimulation rather than simply turning the stimulation completely off.

An ON-ZONE-OFF (ONZOFF) controller was designed to assist sitting down with FNS [18]. Much like the SCC, it used a switching curve in the state space of knee angle and knee angular velocity to determine the state of the stimulation. However, this controller added a "zone" between the on and off states that smoothly increased or decreased stimulation pulse width to the quadriceps and hamstring muscles while keeping stimulation pulse width to the gluteal muscles constant. The ONZOFF controller was compared with an On/Off controller that modulated stimulation to the quadriceps between a maximum $(\mathrm{On})$ or minimum $(\mathrm{Off})$ based on a switching line, rather than a curve, determined by the knee angle versus knee angular velocity profile. The ONZOFF controller reduced mean terminal knee angular velocity by 37 percent when compared with the On/Off controller implemented in the same subjects $(67.6 \pm 33.1 \% \mathrm{~s}$ vs $106.9 \pm 16.7 \%$ s). The authors reported having difficulty selecting the correct switching line for the On/Off controller to handle perturbations or unpredictable shifts in posture or changes in upper-limb forces exerted on a walker during the maneuver. The ONZOFF controller appeared to be more robust and better able to account for perturbations by working within the zone area of the controller that slowly increased or decreased the stimulation pulse width. This was a considerable improvement over the open-loop and On/Off control systems.

Even though the ONZOFF controller reduced terminal knee angular velocity, subjects using FNS still exhibited a much higher velocity than nondisabled individuals, whose knee angular velocity at maximum knee flexion angle was $21.3 \pm 14.0 \%$ [ [17]. In addition, the ONZOFF controller required participants to exert arm forces of approximately 50 percent of body weight $(\% \mathrm{BW})$ on a support device during the maneuver. The vertical acceleration of the body center of mass at the impact and the impact force with the sitting surface were not reported [17-18].

The impact forces that occur when first making contact with the chair have not been previously studied for the STS maneuver with FNS. Chen et al. investigated the influence of posture on sitting impact forces when stroke patients performed STS maneuvers [19]. Sitting impact force ranged between 70 and $80 \% \mathrm{BW}( \pm 28 \%-37 \%)$.

The purpose of our study was to describe and compare the biomechanics of STS in terms of impact forces, upperlimb forces, vertical acceleration at the impact, and knee and hip kinematics in nondisabled volunteers and individuals with SCI sitting down with open-loop ramped stimulation $[2,20]$. Although previous studies found that large upper-limb forces were exerted when sitting down with stimulation and that the motion only began after stimulation was turned off, the forces that occur on the buttocks at initial contact with the chair have not been quantified and warrant investigation, particularly with ramped stimulation. By understanding these forces, we can minimize the risk of injury during STS and determine the desired characteristics of new neuroprosthetic interventions and control systems for more natural STS transitions. 


\section{METHODS}

\section{Participants}

From a power analysis based on the effect size determined from preliminary force and vertical acceleration data, a total of five subjects per population was required to meet statistical significance $(\alpha=0.05, \beta=0.95)$. Five nondisabled male volunteers (subjects $\mathrm{A}-\mathrm{E}$ ) ranging in age from 23 to $63 \mathrm{yr}$ (mean $45 \mathrm{yr}$ ), with a mean weight of $74 \pm$ $6 \mathrm{~kg}$ and height of $170 \pm 23 \mathrm{~cm}$, participated in the study. Similarly, five ( 2 female, 3 male) individuals with chronic SCI (mean time postinjury of $10 \pm 11 \mathrm{yr}$ ) who had received implanted neuroprostheses for standing (mean time postimplant $8 \mathrm{yr}$ ) were recruited. The volunteers with SCI (subjects F-J) ranged in age from 46 to $58 \mathrm{yr}$ (mean $53 \mathrm{yr}$ ), with a mean weight and height of $75 \pm 14 \mathrm{~kg}$ and $174 \pm 11 \mathrm{~cm}$, respectively (Table). There were no significant differences in age $(p=0.42)$, height $(p=0.68)$, and weight $(p=0.84)$ between the nondisabled controls and subjects with SCI. There were also no significant differences in age $(p=0.85)$, height $(p=0.15)$, and weight $(p=$ 0.28 ) between males and females for the subjects with SCI. All subjects signed consent forms approved by the local institutional review board before participation in the study.

\section{Electrical Stimulation Systems}

Subjects using FNS were selected from a variety of projects that historically instrumented different muscle groups for different study purposes. The common muscles stimulated for the STS maneuver in all subjects with SCI, however, included the hip and knee extensor muscles, which are primarily responsible for lifting and lowering the body. Some subjects utilized stimulation of additional muscles to provide added stability in the ante- rior-posterior or medial-lateral directions. Despite the heterogeneity in the number and locations of the implanted electrodes, the muscle sets were not functionally different in their ability to restore the STS maneuver. Stimulation values consistently ramped down over $2 \mathrm{~s}$ across all muscle groups in each subject during the STS maneuver. One subject with SCI (subject F) had 14 percutaneous intramuscular electrodes implanted bilaterally for walking while participating in another project in our laboratory. The electrodes used during standing and the STS maneuver in this study targeted the hip extensors (the posterior portion of the adductor magnus and gluteus maximus) and knee extensors (quadriceps via the femoral nerve). Subject $F$ also utilized individual electrodes in the vastus medialis, intermedius, and lateralis of the quadriceps for additional stability. The other four subjects with SCI (subjects G-J) had received implanted lower-limb neuroprostheses for standing consisting of multichannel implanted pulse generators and intramuscular or spiral nerve cuff electrodes [21-22] to activate the trunk, hip, knee, and/or ankle muscles during participation in other projects in our laboratory. Electrodes targeting stimulation of the hip extensors (gluteus maximus and hamstrings) and knee extensors (quadriceps) were utilized in all subjects with implanted systems. Some subjects $(\mathrm{H}-\mathrm{J})$ also took advantage of the additional stability provided by electrodes in the posterior portion of the adductor magnus to augment hip extension. Subjects G, H, and J also utilized electrodes that activated the trunk extensor (lumbar erector spinae) muscles. The hip abductor (gluteus medius) muscle provided extra stability for subjects $\mathrm{H}$ and I. Subject $\mathrm{H}$ also utilized stimulation of individual heads of the quadriceps (vastus medialis, intermedius, and lateralis) as well as the quadratus lumborum for stabilizing lateral flexion of the trunk. The ankle dorsiflexor

Table.

Characteristics of subjects with spinal cord injury.

\begin{tabular}{lccccccccc}
\hline Subject & Sex & $\begin{array}{c}\text { Age } \\
(\mathbf{y r})\end{array}$ & $\begin{array}{c}\text { Weight } \\
\mathbf{( k g )}\end{array}$ & $\begin{array}{c}\text { Height } \\
\mathbf{( c m )}\end{array}$ & $\begin{array}{c}\text { Injury } \\
\text { Level }\end{array}$ & AIS & $\begin{array}{c}\text { Time Since } \\
\text { Injury (yr) }\end{array}$ & $\begin{array}{c}\text { Time Since } \\
\text { Implant (yr) }\end{array}$ & $\begin{array}{c}\text { Muscles Stimulated } \\
\text { During STS }\end{array}$ \\
\hline F & M & 50 & 67 & 174 & T7 & A & 29 & 29 & QD, GX, VM, VI, VL, PA \\
G & F & 46 & 58 & 168 & T6 & C & 4 & 0.6 & QD, GX, HS, ES \\
H & M & 56 & 84 & 175 & C7 & B & 5 & 1 & QD, GX, HS, PA, GM, ES, QL, \\
& & & & & & & & 2 & VI, VL \\
I & M & 53 & 94 & 192 & T11 & B & 4 & QD, GX, HS, PA, GM \\
J & F & 58 & 74 & 163 & T5 & B & 9 & Q & QD, GX, HS, PA, ES, TA
\end{tabular}

AIS $=$ American Spinal Injury Association Impairment Scale, $\mathrm{C}=$ cervical, $\mathrm{ES}=$ lumbar erector spinae, $\mathrm{F}=$ female, $\mathrm{GM}=$ gluteus medius, $\mathrm{GX}=$ gluteus maximus, $\mathrm{HS}=$ hamstrings, $\mathrm{M}=$ male, $\mathrm{PA}=$ posterior portion of adductor magnus, $\mathrm{QD}=$ quadriceps, $\mathrm{QL}=$ quadratus lumborum, $\mathrm{T}=$ thoracic, $\mathrm{TA}=$ tibialis anterior, $\mathrm{VI}=$ vastus intermedius, $\mathrm{VL}=$ vastus lateralis, $\mathrm{VM}=$ vastus medialis. 
(tibialis anterior) muscle was targeted to counterbalance the plantar flexor contractures during standing that were common for subject J. Subjects with American Spinal Injury Association Impairment Scale (AIS) B injuries exhibited no volitional muscle control that would contribute in the STS maneuver. The volitional function of the hip extensors and knee extensors for subject $\mathrm{G}$, who exhibited an AIS C injury, was determined by a manual muscle test to be grade 1 and grade 2, respectively, and was therefore unable to contribute to body weight support and controlled descent. No stimulation was used with the nondisabled subjects.

\section{Data Collection}

A Vicon ${ }^{\circledR}$ MX40 motion capture system (Vicon Motion Systems; Oxford, UK) tracked the kinematics of the STS maneuvers (Figure 1). Subjects had reflective markers placed bilaterally on their upper body (acromion process, lateral epicondyle, wrist, and third metacarpal), trunk (cervical 7, thoracic 6, anterior and posterior superior iliac spines, and sacrum), and lower body (trochanter, lateral surface of midthigh, lateral epicondyle of the knee, lateral surface of midshank, lateral malleolus, calcaneous, and second metatarsal head). Marker trajectories were collected at $200 \mathrm{~Hz}$. Subjects stood with each foot on a separate biomechanics platform (AMTI; Watertown, Massachusetts) to measure the ground reaction forces as well as to account for all forces occurring dur-

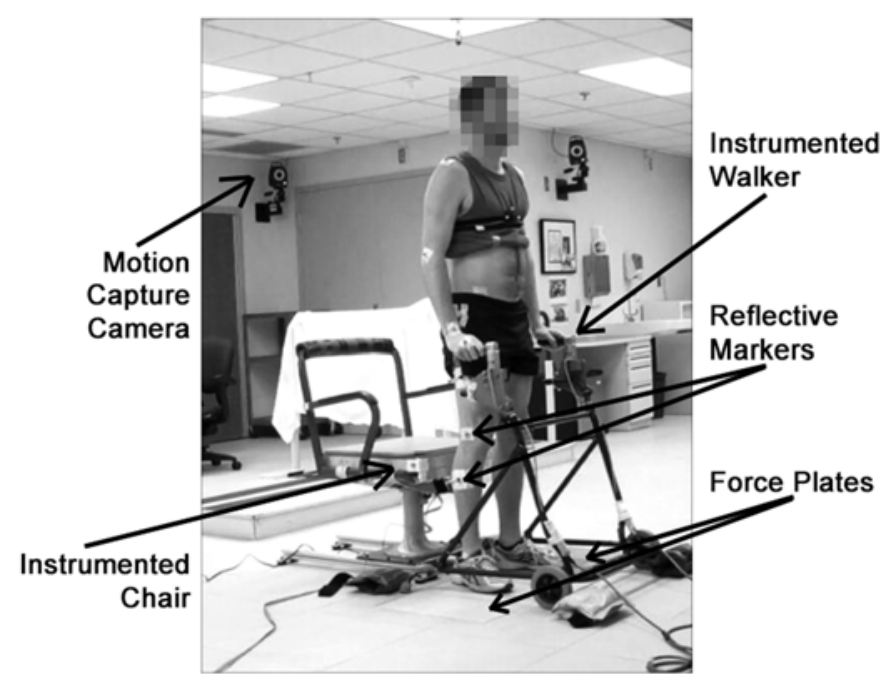

Figure 1.

Experimental setup for nondisabled subject performing standto-sit maneuver. ing the maneuver. To measure the impact force when sitting down, the seat of a chair was instrumented with another force plate. A thin $(1.3 \mathrm{~cm})$ Poron Medical ${ }^{\circledR}$ urethane cushion (Rogers Corporation; Rogers, Connecticut) was placed on top of the force plate to minimize any potential injuries that may occur during the STS maneuver without significantly dampening the impact at contact. Chair height was set to $48 \mathrm{~cm}$, the average popliteal height in American males as per the NASA-STD-3000 Man-Systems Integration Standards [23], resulting in a standardized height for all subjects comparable to a typical wheelchair. The subjects also used a standard walker instrumented with two 6-axis load cells (AMTI) to record the upper-limb forces exerted during STS. The height of the walker handles was adjusted to the preferred height for each subject. Force signals were sampled at 1,000 Hz. The forces and kinematic data were used to calculate kinetics of the movement in postprocessing.

\section{Procedure}

Participants performed at least five trials of the STS maneuver. Subjects began each trial in a standing position with their hands on the instrumented walker and ended each trial in a seated position on the stabilized instrumented chair with their hands on the walker. Nondisabled subjects were instructed to perform the STS maneuver at their preferred comfortable pace while keeping their hands on the walker. Subjects using FNS were instructed to perform the STS maneuver utilizing their preprogrammed, open-loop FNS pattern. This subjectspecific pattern was optimized by a physical therapist and ramped down the pulse width value of the stimulation for the bilateral trunk, hip, knee, and ankle muscles (as listed in the Table) over $2 \mathrm{~s}$ while keeping the frequency $(20 \mathrm{~Hz})$ and amplitude (maximum of $20 \mathrm{~mA}$ for intramuscular electrodes, maximum of $2 \mathrm{~mA}$ for femoral nerve cuff electrodes) constant [22,24-25]. The resting interval between trials was at least $1 \mathrm{~min}$, with the option to rest longer if needed for all participants. All subjects with SCI were spotted by a physical therapist throughout the entire experiment for safety.

\section{Postprocessing}

The data were processed offline. Five trials for each subject (power analysis with $\alpha=0.05, \beta=0.95$ ) were used to determine an ensemble average of the outcome measures. The initiation to complete the STS maneuver was determined differently for nondisabled subjects and 
subjects using FNS. With no repeatable signal to indicate STS intention, the start of the nondisabled maneuver was determined by a deviation of $3^{\circ}$ from the stationary starting knee angle [26]. For subjects using FNS, the beginning of the maneuver was based on the start of the stimulation pattern, where the intended start of the maneuver was indicated by the press of a button that initiated the ramp down of the stimulation. The end of the STS maneuver for all subjects was determined by the peak initial impact force on the chair.

The marker trajectories were labeled and processed using the Vicon Nexus software (Vicon Motion Systems). The kinematic and analog data were processed using a 5th order low pass Butterworth filter with a cutoff frequency of $10 \mathrm{~Hz}$. Ensemble averages for upper-limb force, vertical acceleration at the impact, and impact force for the STS maneuver were calculated. The maximum values in the vertical component of the right and left upper-limb forces for each trial were averaged together to find the peak upper-limb force. Vertical acceleration, or the acceleration at the time of impact, was determined using double differentiation from the trajectory of the sacrum marker and smoothed using a moving average filter $(50 \mathrm{~ms})$. The peak values were determined for the mean upper limb and impact forces and the acceleration at impact during the movement. To compare between subjects, the forces were normalized by body weight.

Data were analyzed utilizing Minitab 16 Statistical Software (Minitab Inc; State College, Pennsylvania). A one-way analysis of variance test with a 95 percent confidence interval $(p<0.05)$ was performed to determine the statistically significant differences between the nondisabled and SCI subject populations, as well as to determine whether there were significant differences within the populations.

\section{RESULTS}

Mean time to complete the STS maneuver differed significantly $(p<0.001)$ between nondisabled subjects $(1.51 \pm 0.45 \mathrm{~s})$ and subjects with SCI using FNS (2.91 \pm $0.36 \mathrm{~s})$. Nondisabled STS involved a simple flexion of the knees and gradual lowering of the body, as can be seen in the lower row of images in Figure 2. Subjects with SCI, on the other hand, had a typical forward leaning posture and continually relied on the walker for upper-body sup- port, as illustrated in the upper row of images in Figure 2. In general, the stimulation began to ramp down before the subjects with SCI initiated the STS, which is approximately Figure 2(a). The SCI subjects leaned forward, shifted the pelvis posteriorly, and flexed at the hip while supporting the upper body with the arms on the walker. This passively locked the knees in extension (Figure 2(b)), thus preventing initiation of the STS maneuver with knee flexion. When the stimulated muscles relaxed sufficiently and the passive extension moment at the knees was reduced by shifting the posture more posteriorly through upper-limb interaction with the walker, the knees were allowed to flex and the subjects began to sit down (Figure 2(c)). Figure 2(d) demonstrates progression of the maneuver by the participants, with the SCI and nondisabled subjects making contact with the chair in Figure 2(e) and 2(f), respectively. The forward lean of SCI subjects until sitting down was completed and an upright seated posture was obtained, as further illustrated in Figure 2(f). When the duration of the maneuver for the SCI subjects was determined in the same way as for the nondisabled subjects (from the time of $3^{\circ}$ knee angle deviation to the impact force), the total time to complete the maneuver was $1.15 \pm 0.43 \mathrm{~s}$. This was significantly faster from the time taken by nondisabled subjects to complete the STS $(p=0.006)$ and essentially represents a descent controlled primarily by upper-limb effort. About 93 percent of the stimulation ramp down (or $1.86 \pm 0.28 \mathrm{~s}$ out of the $2 \mathrm{~s}$ stimulation pattern) had been completed before a change in knee angle for the subjects with SCI occurred.

During the STS maneuver, the knee angle ranged between $0^{\circ}$ (standing position) and $83.4^{\circ} \pm 11.4^{\circ}$ (sitting position) for nondisabled subjects and between $0^{\circ}$ and $85.5^{\circ} \pm 8.03^{\circ}$ for subjects using FNS. The hip angle ranged between $0^{\circ}$ and $77.1^{\circ} \pm 6.5^{\circ}$ for nondisabled subjects and between $0^{\circ}$ and $66.7^{\circ} \pm 9.1^{\circ}$ for subjects using FNS. There was no significant difference in the final knee $(p=0.75)$ and peak hip $(p=0.08)$ angles between the populations. The ending knee angle was influenced primarily by chair height and by the position of the feet relative to the subject's final sitting position. While not controlled in the experimental setup (except for the requirement of one foot per force plate), the position of the feet remained constant for each subject, and both foot and final sitting position varied only slightly between subjects. The trunk, however, was unconstrained and subjects could move their hips and trunk freely as needed 


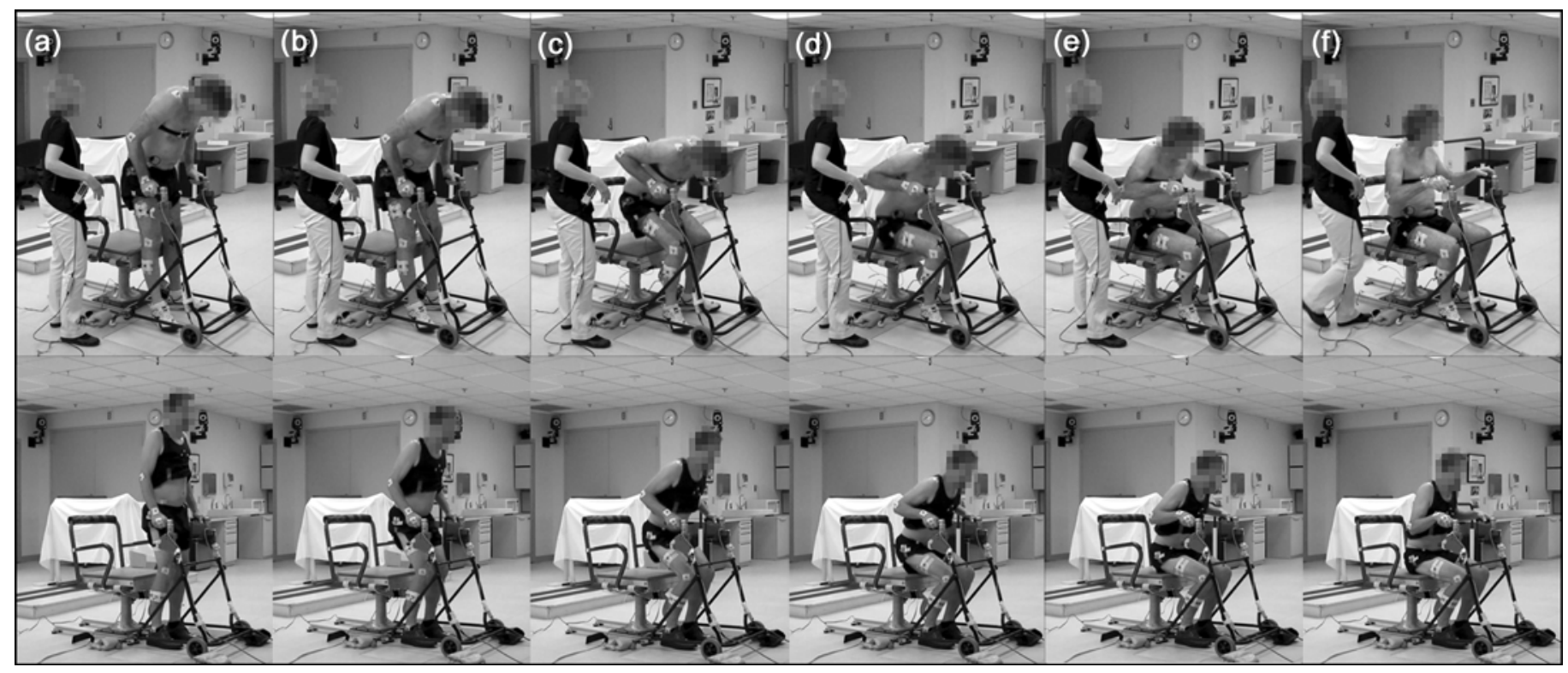

Figure 2.

Typical progression of stand-to-sit (STS) maneuver demonstrated by subject I with spinal cord injury using functional neuromuscular stimulation (FNS) (top row) and nondisabled subject D (bottom row). In example shown, ramping down of stimulation for subject I was initiated $1.8 \mathrm{~s}$ before frame (a), which illustrates only last $10 \%$ of stimulation pattern. Stimulation ended after frame (a) and no further stimulation was applied for frames (b)-(f). Subjects using FNS typically initiated STS maneuver by leaning forward while stimulation was decreasing (a) and exaggerated flexion of hips (b), which led to passive locking of knees even after cessation of stimulation. Once their stimulated muscles were relaxed and posture changed to unlock knees, knees would flex (c) to allow descent toward chair (d)-(f).

during the maneuver by interacting with the walker with their arms, resulting in the large variation in hip angle.

Figure 3 quantitatively shows the hip versus knee angles during STS. In general, as illustrated in Figure 2, the nondisabled STS maneuver exhibited approximately a 1:1 ratio of the hip-to-knee angle during the maneuver. Knee flexion readily took place as needed, proportionally with the hip. On the other hand, the SCI subjects maintained the knees locked in extension with the hips flexed as they leaned forward during the initial portion of the maneuver (this can be related to Figure 2(a)-(b)), resulting in a hip-to-knee angle ratio of approximately 2:1. When the stimulated muscles relaxed and the passive knee extension moment reduced by shifting posture (Figure 2(c)), the knees flexed rapidly between the angles of $20^{\circ}$ to approximately $80^{\circ}$ (Figure 3), while the hips remained flexed at approximately $50^{\circ}$ to $60^{\circ}$ as the subject leaned forward on the walker. The final curl of this graph indicates that the SCI subject was seated and extended the trunk to a more vertical posture, decreasing the hip angle.
The peak knee angular velocity averaged $84.9 \pm 27.0 \% \mathrm{~s}$ across all nondisabled subjects and reached a mean of $166.5 \pm 60.3 \%$ across all subjects using FNS. The knee angular velocity versus knee angle profiles during STS had similar shapes within the subject populations, as represented in Figure 4. The knee angular velocity for subjects using FNS was highly variable, especially beyond $40^{\circ}$ of knee flexion, and was found to be significantly higher than nondisabled values $(p=0.03)$.

\section{Peak Upper-Limb Force}

The peak value of the averaged left and right upperlimb forces normalized by body weight during the STS maneuver after knee angle deviated by $3^{\circ}$ from quiet standing for subjects using FNS $(25.1 \pm 5.1 \% \mathrm{BW})$ was significantly higher $(p<0.001)$ than the nondisabled controls $(7.2 \pm 4.8 \% \mathrm{BW})$, as shown in Figure 5. The peak value of the averaged left and right upper-limb forces during the STS maneuver (as determined by the start of the stimulation ramp down) for subjects using FNS 


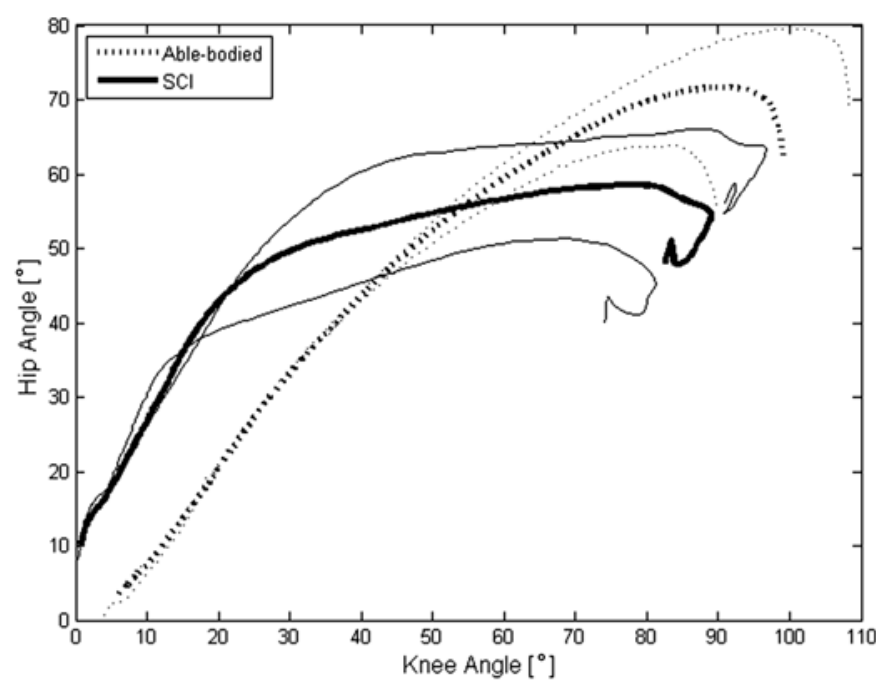

Figure 3.

Representative hip-knee angle plot with standard deviations for nondisabled control (Able-bodied) and subject with spinal cord injury (SCl) using functional neuromuscular stimulation (FNS). Hip and knee angles of nondisabled stand-to-sit (STS) maneuvers approximately follow a 1:1 ratio (dotted line). Subjects using FNS began STS maneuver with large flexion at hips in relation to knees for $\sim 2: 1$ hip-knee angle ratio (thick solid line) but then shifted to rapid change in knee flexion with relatively little change in hip flexion for hip-knee ratio closer to 1:4 in latter part of maneuver.

increased to $27.0 \pm 3.9 \% \mathrm{BW}$ and was not significantly different than the forces measured during the time of the maneuver determined by the change in knee angle. Within the nondisabled population, the peak upper-limb force for subject $\mathrm{C}$ was found to be significantly higher than the other nondisabled subjects, and the upper-limb force for subject $\mathrm{D}$ was found to be significantly less than for subjects B and E. Within the population using FNS, the peak upper-limb force was found to be significantly lower for subject $\mathrm{H}$ than for the other subjects with SCI, and the upper-limb force for subject $\mathrm{F}$ was found to be significantly lower than for subject I. Comparing subjects in the different populations, the peak upper-limb force for nondisabled subject $\mathrm{C}$ was not found to be significantly different than SCI subject $\mathrm{H}$.

\section{Peak Vertical Acceleration}

The peak vertical acceleration at impact was significantly lower $(p<0.001)$ in nondisabled controls $(2.8 \pm$

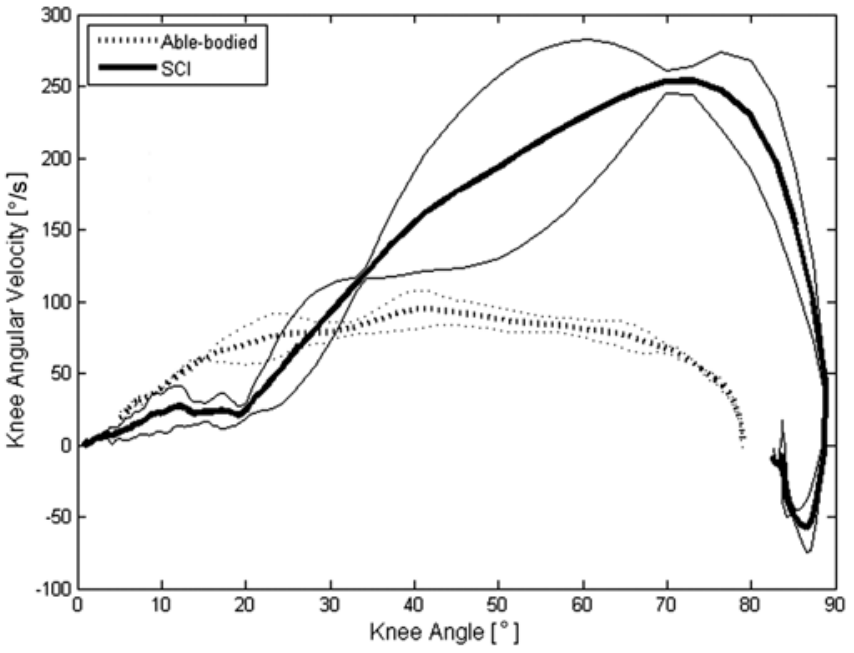

Figure 4.

Representative knee angular velocity with standard deviations for typical nondisabled control (Able-bodied) and subject with spinal cord injury (SCl) using functional neuromuscular stimulation (FNS) during stand-to-sit (STS) maneuver. Knee angular velocity for nondisabled participant was relatively constant throughout STS maneuver (dotted line). Knee angular velocity for subject using FNS increased to maximum in later stages of maneuver (thick solid line) and consistently peaked at values far exceeding those of nondisabled control.

$\left.0.9 \mathrm{~m} / \mathrm{s}^{2}\right)$ than subjects with SCI using FNS $(17.0 \pm$ $\left.4.1 \mathrm{~m} / \mathrm{s}^{2}\right)$, as shown in Figure 6. There was no significant difference within the nondisabled population. However, the vertical acceleration at the impact for SCI subject F was found to be significantly higher than subjects $\mathrm{G}, \mathrm{H}$, and J. The vertical acceleration at the impact for subject $\mathrm{G}$ was found to be significantly lower than subject $\mathrm{I}$, and the vertical acceleration for subject $\mathrm{H}$ was significantly lower than subjects I and J.

\section{Peak Impact Force}

The peak impact forces when the subjects initially made contact with the chair were significantly lower $(p<$ $0.001)$ for nondisabled controls $(71.3 \pm 9.6 \% \mathrm{BW})$ than subjects using FNS $(141.0 \pm 32.1 \% \mathrm{BW})$, as illustrated in Figure 7. Within the nondisabled population, there was no significant difference. There was no significant difference between the impact forces for SCI subjects $\mathrm{G}, \mathrm{H}$, and I. However, the impact force for subject $F$ was found to be significantly higher than subjects $\mathrm{H}$ and $\mathrm{J}$ but not 


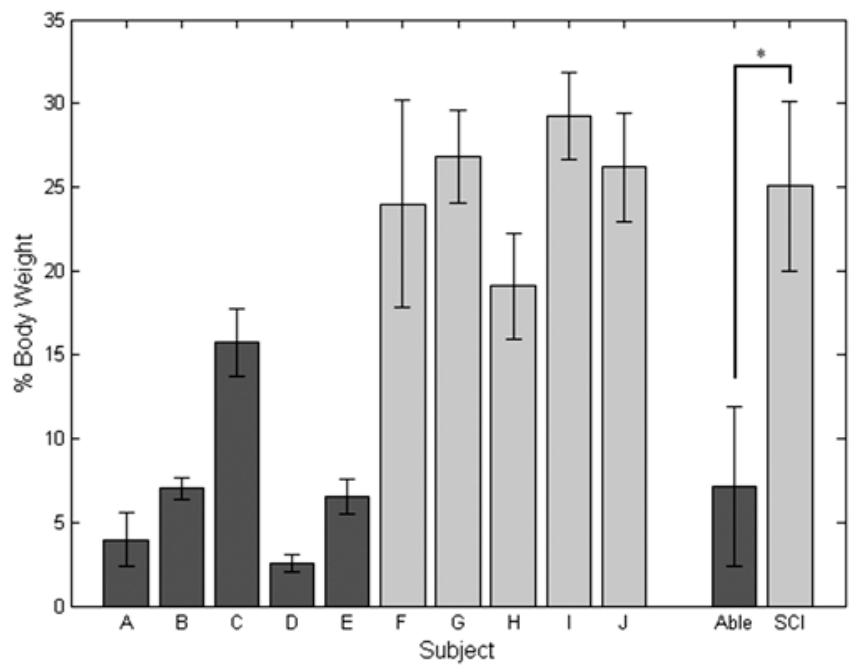

Figure 5.

Mean \pm standard deviation peak upper-limb forces during stand-to-sit maneuver for each subject. Peak values were averaged within nondisabled (Able) and spinal cord injury ( $\mathrm{SCl}$ ) groups to compare between the two populations. ${ }^{*} p<0.05$.

significantly different from subjects $G$ and I. Subject $G$ had an impact force that was significantly higher than subject $\mathrm{J}$.

Because the feet remained in contact with the floor throughout the maneuver, the final forces measured on the instrumented chair for the nondisabled controls were less than the total body weight. When standing, the ground reaction forces for the nondisabled subjects were generally symmetric and ranged between 35 and $50 \%$ BW per leg. There were slight offsets between the right and left legs due to the natural tendency to shift the body weight between legs. On the other hand, subjects using FNS had more asymmetry in the ground reaction forces during standing, which ranged between 15 and $50 \% \mathrm{BW}$ per leg. This asymmetry and larger range of ground reaction forces may be due to differences in muscle strength as generated by the stimulation and lack of proprioception and sensory feedback. It is important to note that the upper-limb forces for the FNS users were symmetric between the right and left arms.

\section{DISCUSSION}

This study provides a comparison of the STS maneuver for nondisabled subjects and individuals with SCI

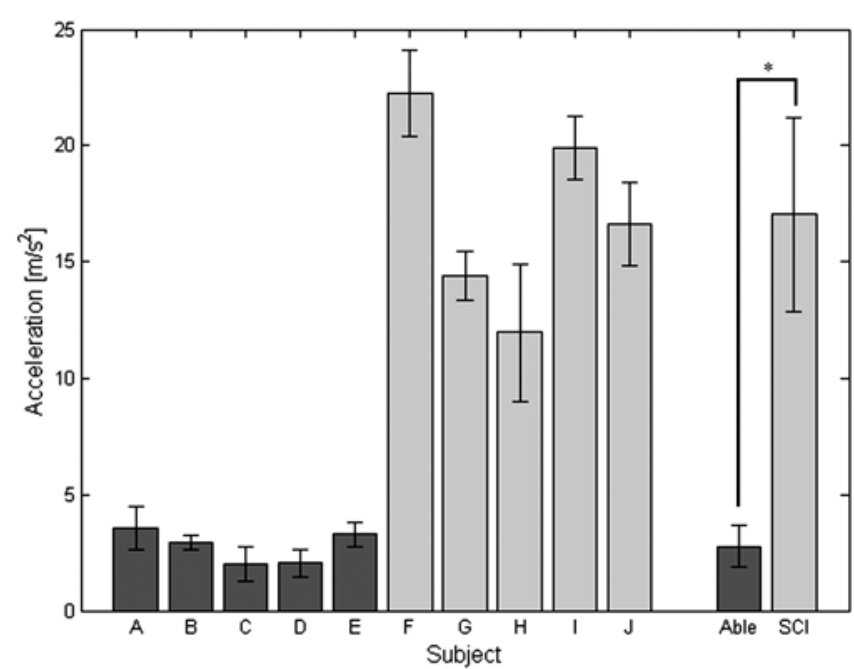

Figure 6.

Mean \pm standard deviation peak vertical accelerations at impact during stand-to-sit maneuver for each subject. Peak values were averaged within nondisabled (Able) and spinal cord injury (SCl) groups to compare between the two populations. ${ }^{*} p<0.05$.

using neuroprostheses with FNS for standing. Use of an open-loop ramp down stimulation pattern for sitting down in individuals with SCI resulted in larger upperlimb forces, vertical acceleration of the sacrum at impact, and sitting impact force as compared with nondisabled controls. Incidents of injury due to a lack of control during STS have not occurred over years of use by the numerous subjects implanted. The stimulation pattern used for the STS maneuver needs to be improved and optimized to better emulate nondisabled STS. Doing so would allow descending onto a seating surface that may be harder or less cushioned than the user's wheelchair, and the maneuver could be completed with less conscious or upper-limb effort. The time to complete the descent phase of the STS as measured from the initial change in knee angle was about the same for both subject populations. However, subjects with SCI tended to precede knee flexion with a posterior pelvic shift and hip flexion at the start of the ramp down pattern, which effectively may have resulted in a longer time to complete the entire STS.

Because the open-loop ramp down of stimulus pulse duration to the hip and knee extensor muscles was almost complete when the knees of the SCI subjects began to flex, a majority of the downward movement of the body was not 


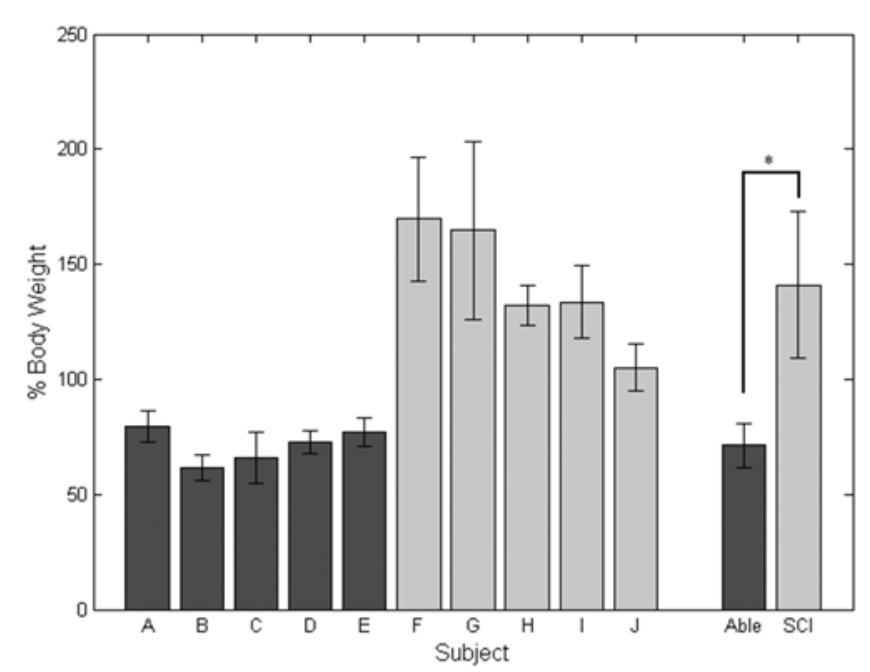

Figure 7.

Mean \pm standard deviation peak impact forces during stand-tosit maneuver for each subject. Peak values were averaged within nondisabled (Able) and spinal cord injury ( $\mathrm{SCl}$ ) groups to compare between the two populations. ${ }^{*} p<0.05$.

resisted by eccentric contractions of the stimulated muscles but instead was controlled primarily by the upper limbs to slow descent. Subjects with SCI relied heavily on their upper limbs to support themselves throughout the maneuver, as indicated by the peak upper-limb forces that averaged 3.5 times (ranging between 1.7 and 12 times) those of nondisabled controls. Repetitive high peak forces on the upper limbs for individuals with SCI can lead to pain in the shoulders, an inability to push a manual wheelchair, and a lower quality of life [27-28]. Nondisabled subjects do not typically require use of their hands to sit down. The upperlimb forces measured for nondisabled controls were merely the weights of their arms resting passively on the instrumented walker and, as such, did not indicate a reliance on the upper limbs for support. Although subject $C$ exerted a larger peak upper-limb force than the other nondisabled subjects, he did not exhibit any medical conditions that could cause difficulty in completing the maneuver. Despite the relatively high values for subject C, all of the nondisabled subjects' upper-limb forces were still lower than those of the FNS users who relied on their arms for support. There was a difference in the peak upper-limb force between subject $\mathrm{H}$ and the other FNS users; however, it was not found to correlate with either a higher impact force or peak vertical acceleration compared with the other subjects with SCI. Stimulation patterns were comparable, and there were no significant differences between subjects of different sexes in height, weight, or time postinjury.

The vertical acceleration of the center of mass at impact for those using FNS was on average 6 times (and between 3.5 and 11 times) higher than that of nondisabled subjects. The acceleration measured at impact for the subjects with SCI reached almost $2 g$. While it would only be possible to reach a maximum acceleration of $1 g$ (the acceleration due to gravity) during free fall, the acceleration at the impact is technically a deceleration because the body is being slowed by the contact with the chair and can become higher than $1 g$. Snyder et al. performed a study on the impact tolerance of the human body from free fall events [29]. The peak resultant accelerations of the pelvis at the impact after free falls from 5 and $8 \mathrm{ft}$ in that study ranged between 104 and $193 g$. The high accelerations at impact in that study verify the validity of the peak acceleration values we measured at impact during STS.

Impact forces for subjects using FNS were 1.3 to 2.8 times higher than for nondisabled subjects and approximately 1.1 to 1.7 times their own body weight. The nondisabled subjects are capable of controlling the eccentric contractions of their STS muscles [15-16], primarily the quadriceps, and utilize the resistive torque created by that contraction to slow their descent enough during the maneuver to experience a softer landing. This resulted in impact forces that were less than total body weight. Because the subjects' feet remained in contact with the ground during the entire maneuver, the weight of the legs was not included in the force measurement on the chair. Nondisabled subjects typically sit without arm support in a real-world environment but were asked to keep their hands on the instrumented walker during the maneuver in order to control for the potential influence of the assistive device used by the subjects with SCI. Using the walker would reduce the impact forces of the nondisabled participants, thereby minimizing differences with FNS users, whereas not using the walker would have made the maneuver impossible for the subjects with SCI. The experimental conditions used were therefore considered to be conservative. Yet, a significant difference in the impact force was still observed between the two subject groups.

It should be noted that the peak values for upper-limb force, vertical acceleration at impact, and sitting impact force may not have necessarily occurred at the same time 
during the STS maneuver. For example, the peak vertical acceleration at impact does not occur exactly when the peak upper-limb force occurs. In addition, the timing in the peak values may differ between subjects. In particular, the peak upper-limb force may occur early in the maneuver as individuals with SCI lean forward, while peak impact force by definition occurs later in the STS transition during seat contact. For this reason and to facilitate comparisons, peak arm forces were calculated in subjects with SCI only after their knee flexion angles deviated by $3^{\circ}$ from standing, similar to the criteria used to determine the initiations of STS for nondisabled subjects. Nevertheless, peak arm forces with FNS far exceed those exhibited by nondisabled subjects.

Experiencing repeated high impact forces and accelerations at contact during STS is not ideal for neuroprosthesis users who lack normal sensation and should be further investigated. Although our subjects showed no signs of injury or discomfort due to repeated performance of the STS maneuver, animal studies using anesthetized domestic pigs investigated the immediate skin reactions after minor trauma and found deep muscular hemorrhaging to be more prominent in areas with a thin muscular layer when the vasculature was crushed by the underlying bone [30]. No specific thresholds or minimum velocities needed to cause hemorrhaging, or skin or deep tissue damage, were reported. For sitting down, the ischial tuberosity is the bony prominence that could potentially injure the nearby vasculature and cause hemorrhaging or skin damage in response to excessive or repeated impact.

The position of assistive devices, such as a walker, when sitting down may have influenced the inability to slow the maneuver using upper-limb strength. Nondisabled individuals have the option to place one or both of their hands on the chair armrests behind them to help control and steady the rate of descent while sitting down to minimize impact. That strategy is unavailable to subjects standing with FNS who rely on a walker in front of them to provide stability and to maneuver themselves into a good standing posture. Neuroprosthesis users need to push down on the walker with their upper limbs to move the trunk into an upright position. Moving one hand from the walker to place on an armrest behind their back can lead to instability and an increased risk of falling.

Further observation of the STS maneuver with FNS revealed that the knees were locking as subjects prepared to start their descent. While standing, subjects with SCI were able to position themselves into a good, upright, and vertical standing posture, but consistently leaned forward and flexed at their hips as they prepared to begin to descend toward the chair. By the time the knees unlocked, the stimulation pattern was on average 93 percent complete and had nearly ramped completely off. Stimulating the knee flexors when beginning STS could potentially overcome the passive locking that occurs. This locking of the knees may not have allowed the users to descend slowly by gradually flexing the knees as the stimulation pattern ramped down and was likely to have contributed to both the high knee flexion angular velocities observed toward the end of the maneuver and high impact at contact with the chair.

Some of the limitations of this study include the relatively small sample size. Within the SCI population, there is also variation in injury level and sensation, upper-body strength, muscle set with implanted electrodes, and stimulation parameters. Although a power analysis was performed to determine the number of subjects and trials to be performed, a larger sample of nondisabled and SCI subjects would extend the generalizability of the results. A standard height was also selected for the chair, although the height of the subjects varied. Previous studies found that an elevated chair height reduces the angular displacements [31] and angular velocities [32] at the lower limbs in nondisabled subjects during sit-to-stand. In future studies, this potential source of variability could be controlled by individually adjusting chair height for each subject. Elevating chair height could also be a strategy for mitigating impact force and rate of descent during STS.

\section{CONCLUSIONS}

The current investigation demonstrated significantly greater peak values for upper-limb force, impact vertical acceleration, and impact force during the STS maneuver for FNS users than for nondisabled subjects. For subjects performing the STS maneuver using FNS, it is advised that a sufficiently thick protective cushion be provided on the seat to avoid soft tissue injury until the STS is better controlled. Further improvements in the control of stimulation and neuroprosthesis design are needed to normalize the STS maneuver. However, it still may not be possible to control the eccentric contractions of the primary muscles needed during sitting solely using FNS. Future work will involve closed-loop control of knee extensors, adding knee flexion to the stimulation pattern to reduce the 
passive locking of the knees in the beginning of the STS maneuver, or designing an external orthosis with knee damping for use in combination with FNS to provide a safe, controlled STS maneuver. Contracting paralyzed muscles with FNS in individuals with SCI has the potential to significantly improve quality of life by providing opportunity to exercise and options for performing numerous standing functions and transfers [20,33]. FNS users are satisfied with their implanted neuroprotheses, feel healthier because of using them, would undergo the implantation surgery and rehabilitation again for the same result, and would recommend them to a friend [34]. Usage patterns for implanted standing neuroprotheses remain constant and actually shift from exercise to functional standing over the course of the first year postdischarge [2], when most conventional long leg braces are abandoned [35-36]. An implanted system can also be more intimate and conducive to spontaneous use than donning and doffing conspicuous external orthoses multiple times throughout the day. The potential functional outcomes and cost-benefit analyses when combining FNS with orthotic devices or wheelchair standing devices could also be evaluated in future studies designed to improve the control of the STS maneuver.

\section{ACKNOWLEDGMENTS}

\author{
Author Contributions: \\ Study concept and design: R. Kobetic, R. J. Triolo, S. R. Chang. \\ Acquisition of data: S. R. Chang. \\ Analysis and interpretation of data: S. R. Chang, R. Kobetic, \\ R. J. Triolo. \\ Drafting of manuscript: S. R. Chang. \\ Critical revision of manuscript for important intellectual content: \\ R. Kobetic, R. J. Triolo. \\ Statistical analysis: S. R. Chang. \\ Obtained funding: R. Kobetic, R. J. Triolo. \\ Study supervision: R. Kobetic, R. J. Triolo.
}

Financial Disclosures: The authors have declared that no competing interests exist.

Funding/Support: This material was based on work supported by Department of Veterans Affairs, Rehabilitation Research and Development Service (grant B0608-R). S. R. Chang was also supported in part by U.S. Department of Education grant GAANN P200A100112 to the Department of Biomedical Engineering, Case Western Reserve University.

Additional Contributions: The authors would like to thank the subjects for their participation, as well as Kevin Foglyano and Stephanie Nogan Bailey for their technical support and Lisa Lombardo, PT, for her expertise and assistance in preparing and conducting the experiments.
Institutional Review: All volunteers signed consent forms approved by the local institutional review board.

Participant Follow-Up: The authors plan to inform participants of the publication of this study.

\section{REFERENCES}

1. Mushahwar VK, Jacobs PL, Normann RA, Triolo RJ, Kleitman N. New functional electrical stimulation approaches to standing and walking. J Neural Eng. 2007;4(3): S181-97.

[PMID:17873417]

http://dx.doi.org/10.1088/1741-2560/4/3/S05

2. Triolo RJ, Bailey SN, Miller ME, Rohde LM, Anderson JS, Davis JA Jr, Abbas JJ, DiPonio LA, Forrest GP, Gater DR Jr, Yang LJ. Longitudinal performance of a surgically implanted neuroprosthesis for lower-extremity exercise, standing, and transfers after spinal cord injury. Arch Phys Med Rehabil. 2012;93(5):896-904. [PMID:22541312] http://dx.doi.org/10.1016/j.apmr.2012.01.001

3. Marsolais EB, Kobetic R. Development of a practical electrical stimulation system for restoring gait in the paralyzed patient. Clin Orthop Relat Res. 1988;(233):64-74.

[PMID:3261221]

4. Kobetic R, Marsolais EB. Synthesis of paraplegic gait with multichannel functional neuromuscular stimulation. IEEE Trans Rehabil Eng. 1994;2(2):66-79. http://dx.doi.org/10.1109/86.313148

5. Gallien P, Brissot R, Eyssette M, Tell L, Barat M, Wiart L, Petit H. Restoration of gait by functional electrical stimulation for spinal cord injured patients. Paraplegia. 1995; 33(11):660-64. [PMID:8584301] http://dx.doi.org/10.1038/sc.1995.138

6. Nash MS, Jacobs PL, Montalvo BM, Klose KJ, Guest RS, Needham-Shropshire BM. Evaluation of a training program for persons with SCI paraplegia using the Parastep 1 ambulation system: Part 5. Lower extremity blood flow and hyperemic responses to occlusion are augmented by ambulation training. Arch Phys Med Rehabil. 1997;78(8): 808-14. [PMID:9344298] http://dx.doi.org/10.1016/S0003-9993(97)90192-1

7. Graupe D, Kohn KH. Functional neuromuscular stimulator for short-distance ambulation by certain thoracic-level spinal-cord-injured paraplegics. Surg Neurol. 1998;50(3): 202-7. [PMID:9736079] http://dx.doi.org/10.1016/S0090-3019(98)00074-3

8. Guest RS, Klose KJ, Needham-Shropshire BM, Jacobs PL. Evaluation of a training program for persons with SCI paraplegia using the Parastep 1 ambulation system: Part 4. Effect on physical self-concept and depression. Arch Phys Med Rehabil. 1997;78(8):804-7. [PMID:9344297] http://dx.doi.org/10.1016/S0003-9993(97)90191-X 
9. Kralj A, Jaeger RJ, Munih M. Analysis of standing up and sitting down in humans: Definitions and normative data presentation. J Biomech. 1990;23(11):1123-38. [PMID:2277047] http://dx.doi.org/10.1016/0021-9290(90)90005-N

10. Jaeger RJ. Design and simulation of closed-loop electrical stimulation orthoses for restoration of quiet standing in paraplegia. J Biomech. 1986;19(10):825-35.

[PMID:3782165]

http://dx.doi.org/10.1016/0021-9290(86)90133-8

11. Moynahan M, Mullin C, Cohn J, Burns CA, Halden EE, Triolo RJ, Betz RR. Home use of a functional electrical stimulation system for standing and mobility in adolescents with spinal cord injury. Arch Phys Med Rehabil. 1996; 77(10):1005-13. [PMID:8857878] http://dx.doi.org/10.1016/S0003-9993(96)90060-X

12. Abbas JJ, Gillette JC. Using electrical stimulation to control standing posture. IEEE Control Systems. 2001;21(4): 80-90. http://dx.doi.org/10.1109/37.939946

13. Popovic D, Tomović R, Schwirtlich L. Hybrid assistive system - the motor neuroprosthesis. IEEE Trans Biomed Eng. 1989;36(7):729-37. [PMID:2787281] http://dx.doi.org/10.1109/10.32105

14. Nene AV, Jennings SJ. Hybrid paraplegic locomotion with the ParaWalker using intramuscular stimulation: A single subject study. Paraplegia. 1989;27(2):125-32.

[PMID:2785668]

http://dx.doi.org/10.1038/sc.1989.19

15. Ashford S, De Souza L. A comparison of the timing of muscle activity during sitting down compared to standing up. Physiother Res Int. 2000;5(2):111-28.

[PMID:10863717]

http://dx.doi.org/10.1002/pri.190

16. Ferrante S, Pedrocchi A, Ferrigno G. Electromyographic analysis of standing up and sitting down. Proceedings of the International Functional Electrical Stimulation Society Conference; 2005 Jul; Montreal, Canada. Milano (Italy): IFESS; 2005.

17. Dolan MJ, Andrews BJ, Veltink PH. Switching curve controller for FES-assisted standing up and sitting down. IEEE Trans Rehabil Eng. 1998;6(2):167-71. [PMID:9631324] http://dx.doi.org/10.1109/86.681182

18. Poboroniuc MS, Wood DE, Riener R, Donaldson NN. A new controller for FES-assisted sitting down in paraplegia. Adv Electr Comp Eng. 2010;10(4):9-16. http://dx.doi.org/10.4316/aece.2010.04002

19. Chen HB, Wei TS, Chang LW. Postural influence on Standto-Sit leg load sharing strategies and sitting impact forces in stroke patients. Gait Posture. 2010;32(4):576-80. [PMID:20829046] http://dx.doi.org/10.1016/j.gaitpost.2010.08.005
20. Davis JA Jr, Triolo RJ, Uhlir J, Bieri C, Rohde L, Lissy D, Kukke S. Preliminary performance of a surgically implanted neuroprosthesis for standing and transferswhere do we stand? J Rehabil Res Dev. 2001;38(6):609-17. [PMID:11767968]

21. Memberg WD, Peckham PH, Keith MW. A surgically implanted intramuscular electrode for an implantable neuromuscular stimulation system. IEEE Trans Rehabil Eng. 1994;2:80-91. http://dx.doi.org/10.1109/86.313149

22. Fisher LE, Miller ME, Bailey SN, Davis JA Jr, Anderson JS, Rhode L, Tyler DJ, Triolo RJ. Standing after spinal cord injury with four-contact nerve-cuff electrodes for quadriceps stimulation. IEEE Trans Neural Syst Rehabil Eng. 2008;16(5):473-78. [PMID:18990650] http://dx.doi.org/10.1109/TNSRE.2008.2003390

23. National Aeronautics and Space Administration. NASASTD-3000 Man-Systems Integration Standards, Volume 1, Section 1 [Internet]. Houston (TX): NASA; 2000. Available from: http://msis.jsc.nasa.gov/sections/section03.htm

24. Popovic D, Gordon T, Rafuse VF, Prochazka A. Properties of implanted electrodes for functional electrical stimulation. Ann Biomed Eng. 1991;19(3):303-16.

[PMID:1928872]

http://dx.doi.org/10.1007/BF02584305

25. Bhadra N, Kilgore KL, Peckham PH. Implanted stimulators for restoration of function in spinal cord injury. Med Eng Phys. 2001;23(1):19-28. [PMID:11344004] http://dx.doi.org/10.1016/S1350-4533(01)00012-1

26. Yoshioka S, Nagano A, Himeno R, Fukashiro S. Computation of the kinematics and the minimum peak joint moments of sit-to-stand movements. Biomed Eng Online. 2007;6(1):26. [PMID:17608922] http://dx.doi.org/10.1186/1475-925X-6-26

27. Kemp BJ, Bateham AL, Mulroy SJ, Thompson L, Adkins $\mathrm{RH}$, Kahan JS. Effects of reduction in shoulder pain on quality of life and community activities among people living long-term with SCI paraplegia: A randomized control trial. J Spinal Cord Med. 2011;34(3):278-84.

[PMID:21756566] http://dx.doi.org/10.1179/107902611X12972448729486

28. Samuelsson KA, Tropp H, Gerdle B. Shoulder pain and its consequences in paraplegic spinal cord-injured, wheelchair users. Spinal Cord. 2004;42(1):41-46. [PMID:14713943] http://dx.doi.org/10.1038/sj.sc.3101490

29. Snyder RG, Foust DR, Bowman BM. Study of impact tolerance through free-fall investigation. Ann Arbor (MI): Highway Safety Research Institute, University of Michigan; 1977.

30. Randeberg LL, Winnem AM, Langlois NE, Larsen EL, Haaverstad R, Skallerud B, Haugen OA, Svaasand LO. Skin changes following minor trauma. Lasers Surg Med. 2007; 
39(5):403-13. [PMID:17523178]

http://dx.doi.org/10.1002/1sm.20494

31. Rodosky MW, Andriacchi TP, Andersson GB. The influence of chair height on lower limb mechanics during rising. J Orthop Res. 1989;7(2):266-71. [PMID:2918425] http://dx.doi.org/10.1002/jor.1100070215

32. Schenkman M, Riley PO, Pieper C. Sit to stand from progressively lower seat heights - alterations in angular velocity. Clin Biomech (Bristol, Avon). 1996;11(3):153-58. [PMID:11415613] http://dx.doi.org/10.1016/0268-0033(95)00060-7

33. Rohde LM, Bonder BR, Triolo RJ. Exploratory study of perceived quality of life with implanted standing neuroprostheses. J Rehabil Res Dev. 2012;49(2):265-78. [PMID:22773528] http://dx.doi.org/10.1682/JRRD.2010.08.0156

34. Agarwal S, Triolo RJ, Kobetic R, Miller M, Bieri C, Kukke S, Rohde L, Davis JA Jr. Long-term user perceptions of an implanted neuroprosthesis for exercise, standing, and transfers after spinal cord injury. J Rehabil Res Dev. 2003;40(3): 241-52. [PMID:14582528]

35. Phillips B, Zhao H. Predictors of assistive technology abandonment. Assist Technol. 1993;5(1):36-45.
[PMID: 10171664]

http://dx.doi.org/10.1080/10400435.1993.10132205

36. Batavia AI, Hammer GS. Toward the development of consumer-based criteria for the evaluation of assistive devices. J Rehabil Res Dev. 1990;27(4):425-36. [PMID:2089152] http://dx.doi.org/10.1682/JRRD.1990.10.0425

Submitted for publication December 17, 2013. Accepted in revised form July 31, 2014.

This article and any supplementary material should be cited as follows:

Chang SR, Kobetic R, Triolo RJ. Understanding standto-sit maneuver: Implications for motor system neuroprostheses after paralysis. J Rehabil Res Dev. 2014; 51(9):1339-52.

http://dx.doi.org/10.1682/JRRD.2013.12.0264

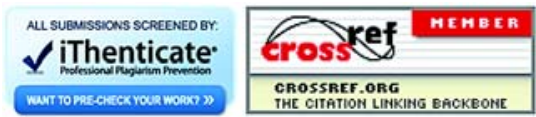


